\title{
Stratégie de prévention : l'aménagement de la Loire à Brives-Charensac
}

\author{
par H. Dumay
}

Directeur du développement Eau-BCEOM

\section{INTRODUCTION}

L'aménagement de la Loire à Brives-Charensac constitue l'une des premières actions d'envergure engagées par l'Etat dans le cadre du plan « Loire Grandeur Nature ».

Les travaux actuellement en cours de réalisation correspondent à un choix technique qui associe protection contre les crues et valorisation du site, c'est-à-dire à une volonté de mettre en harmonie l'homme et la nature rassemblés dans l'environnement du fleuve et de ses abords.

Pour ce faire, il convenait de réaliser un principe de protection contre les crues qui soit compatible avec la réhabilitation du milieu.

L'amélioration des écoulements sans endiguement du fleuve constitue l'idée directrice de ce projet qui, en autorisant toute possibilité d'aménagement, favorise le développement de nouvelles relations entre la ville et le fleuve.

\section{II. - L'HISTORIQUE DU PROJET ET SON ABOUTISSEMENT}

Les principes d'aménagement qui ont guidé ce projet n'ont pas été de tout temps la préoccupation essentielle de l'homme, la rivière étant utilisée pour ses fonctions d'usage les plus directes liées au prélèvement d'eau et à la force hydraulique. En effet, il s'est passé de nombreuses années sans crues catastrophiques, ce qui a conduit à coloniser peu à peu le fleuve et ses abords jusqu'au jour où celui-ci a repris brutalement ses droits, balayant tout sur son passage, rendant dérisoire certains aménagements de protection inadaptés.

C'est ce qui se passe le 21 septembre 1980, lorsqu'une crue brutale du haut bassin de la Loire générée par des pluies diluviennes de plus de $600 \mathrm{~mm}$ en 14 heures, provoque une montée du fleuve de près de 7 mètres à BrivesCharensac en quelques heures. Sur ce court intervalle, le fleuve passe de quelques mètres cubes par seconde à $2000 \mathrm{~m}^{3} / \mathrm{s}$ dévastant tout sur son passage ! Le bilan est catastrophique : 8 morts, 1350 personnes sinistrées, 560 maisons inondées, de nombreuses infrastructures endommagées... Il fallait trouver une solution à l'échelle du problème.
Dans un premier temps, il est envisagé l'édification du barrage de Serre-de-la-Fare situé sur le bassin amont qui permet de laminer efficacement les crues. Cette solution jugée pénalisante par son impact environnemental sur l'emprise même de la retenue est abandonnée.

Le 4 janvier 1994, le Gouvernement décide la mise en œuvre du Plan « Loire Grandeur Nature ». Celui-ci confirme l'abandon du projet de barrage de Serre-de-la-Fare au profit d'un aménagement spécifique destiné à améliorer l'écoulement des eaux dans la traversée de Brives-Charensac. Dès lors, la Direction Départementale de l'Equipement de la Haute-Loire, en liaison avec la Direction de l'Eau du Ministère de l'Environnement propose que cet aménagement se fonde non seulement sur l'objectif sécurité mais puisse également apporter un plus en terme d'image et de valorisation économique du territoire.

Cette stratégie se réfere ainsi aux objectifs et aux ambitions affichés du plan "Loire Grandeur Nature " et, de manière plus générale à l'aménagement du territoire.

Dès le mois de juin 1994, un concours de maîtrise d'œuvre à l'échelle européenne est engagé ; trois équipes, toutes constituées d'un bureau d'études spécialisé en hydraulique, d'architectes de renommée et d'agences de communication ont été désignées début juillet 1994 pour concourir sur les bases d'un cahier des charges alliant les objectifs de sécurité cités plus haut et les objectifs environnementaux.

Ces équipes remettent en octobre 1994 trois projets de natures différentes qui font l'objet d'une exposition publique sur la Commune de Brives-Charensac qui durera 10 jours et qui accueillera et sensibilisera un public important.

Il est à noter la particularité de cette démarche de communication assortie d'une exposition à grande échelle avec plans cartographiques, photomontages, plans architecturaux, plans types schémas directeurs, plans montrant les liens entre le fleuve et la vie de la cité associés aux différents vecteurs d'animation...

Ces documents montrent clairement le risque lié aux crues en situation actuelle, la situation après travaux d'aménagement, le parti d'aménagement retenu dans le cadre d'une relation entre la ville et le fleuve.

C'est donc en toute connaissance de cause et après expertise technique que la commission de jugement composée de l'Administration, d'élus et de techniciens a désigné le lauréat du concours : le groupement BCEOM. 
Ce groupement comprend le BCEOM pour la conception hydraulique et la coordination du projet, le Cabinet CantalDupart pour les aspects architecturaux, Médiacité pour la communication.

\section{III. [ LE CONCEPT D'AMÉNAGEMENT : « CONCILIER SÉCURITÉ, NATURE, ENVIRONNEMENT ET ÉCONOMIE »}

Le projet est conçu à partir de deux objectifs incontournables.

Le premier objectif est une contrainte technique liée à l'objectif sécurité absolue.

Pour une crue centennale du type de celle de septembre 1980, l'aménagement devra garantir la sécurité des riverains, c'est-à-dire une hauteur de moins de 1 mètre d'eau et une vitesse de moins de $1 \mathrm{~m} / \mathrm{s}$.

Deux solutions se présentaient aux techniciens :

- L'isolement du fleuve par endiguement ; il permettait d'assurer une mise hors d'eau totale en arrière des digues pour une crue du type de 1980 et allait donc au-delà des objectifs de protection. Toutefois, ce type d'aménagement constituait une protection du «tout ou rien », c'est-à-dire que pour une crue supérieure à la crue de projet, on risquait d'avoir une submersion brutale et catastrophique d'une hauteur équivalente à la hauteur de la digue en arrière de la zone à protéger.

L'effet d'un tel aménagement s'avérait d'autant plus grave que la présence de la digue procurerait un sentiment de sécurité qui désensibiliserait les riverains sur les risques graves encourus.

Dès le départ, nous avons donc proscrit ce type d'aménagement, les catastrophes les plus récentes illustrant bien l'inadéquation de ces dispositifs par le faux sentiment de sécurité qu'ils génèrent et par la précarité de la protection qu'ils assurent.

- Le rabaissement des niveaux de crues par l'aménagement du lit et ses abords.
Deux actions ont été envisagées :

- dérocter le fond du lit pour supprimer les seuils naturels qui, souvent associés aux ouvrages, constituent un obstacle important à l'écoulement.

Le point noir le plus caractéristique de cette situation est celui du pont de Gallard au centre de Brives-Charensac. Il existe en ce point un véritable goulet d'étranglement qui limite notoirement la capacité de transit par l'ouvrage luimême implanté de surcroît sur un haut fond rocheux.

Il convenait donc, sans changer le profil d'équilibre du lit, d'araser les points hauts du lit mineur que le cours d'eau n'avait pu, au fil du temps, éroder naturellement.

- Modeler les terrains en bordure du fleuve de façon à favoriser une mise en eau lente et progressive assurant par ailleurs un meilleur écoulement des crues exceptionnelles.

Ces deux actions combinées qui assurent un rabaissement efficace des lignes d'eau en crue de 1 à $2,50 \mathrm{~m}$ sur les zones sensibles à protéger ont donc été retenues (fig. l).

Le second objectif correspond à une stratégie d'aménagement qui doit conduire à ouvrir la ville vers le fleuve, de façon à favoriser une valorisation urbaine et économique du site.

Bien entendu, cette action a été particulièrement favorisée par le principe même de l'aménagement hydraulique qui ne créait aucune " barrière ", aucun effet de coupure.

Ces conditions étaient particulièrement favorables pour "réconcilier " la ville avec le fleuve, nouer de nouvelles relations en facilitant l'accès et l'attractivité du fleuve et de ses abords par aménagement.

D'une façon plus générale, le projet que nous allons décrire plus précisément présente l'avantage de conjuguer protection contre les crues, respect et valorisation de l'environnement.

L'aspect novateur de ce concept d'aménagement a pu prendre sa pleine expression parce que la réflexion a été menée par une équipe pluridisciplinaire élargie à tous les acteurs locaux.

Dans ce contexte, rien ne s'oppose désormais à une appropriation du projet par les riverains de façon à le faire vivre dans le cadre d'une Loire respectée et respectueuse de l'homme.

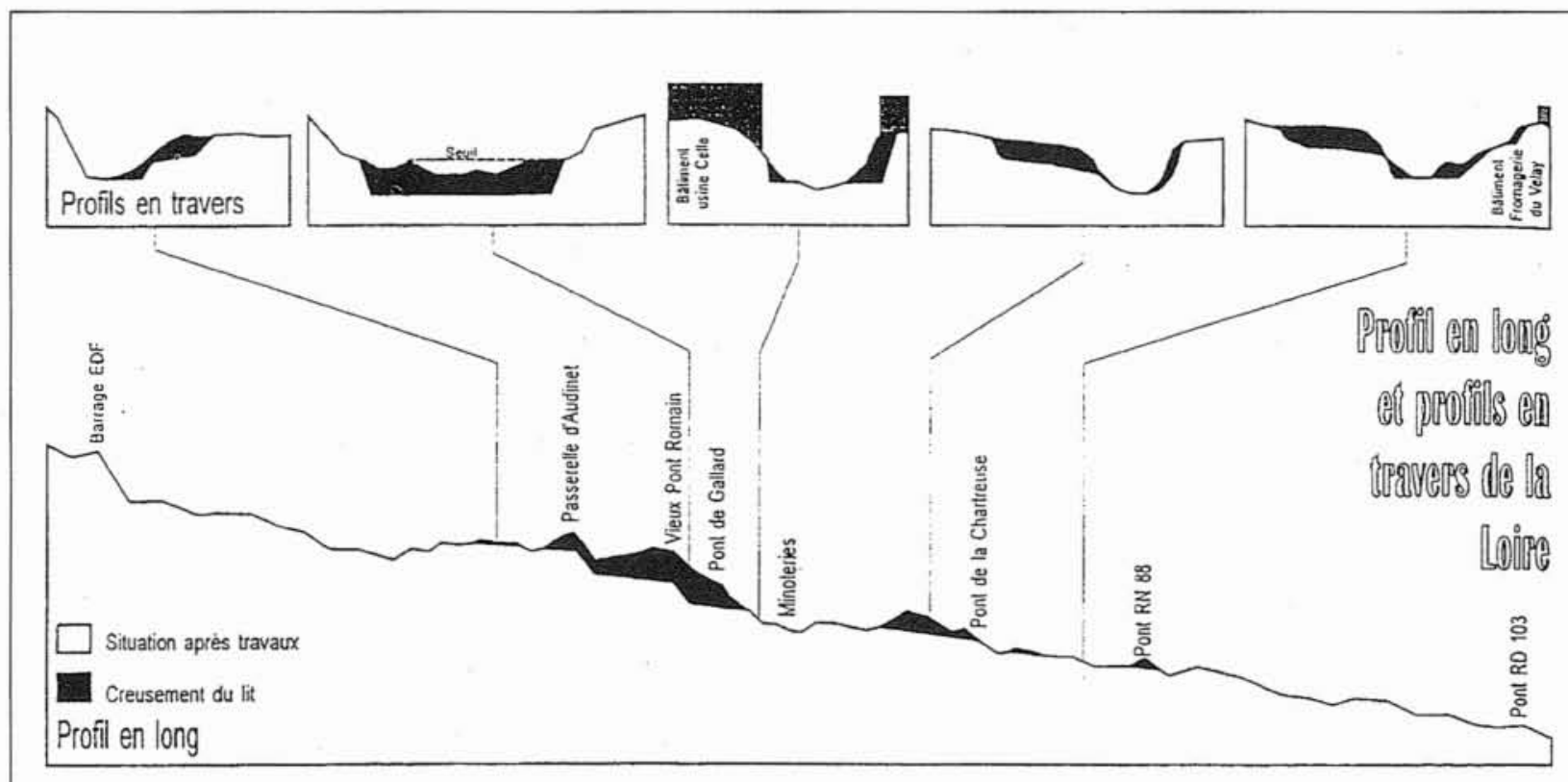

1. Profil en long et profils en travers de la Loire. 


\section{IL PROJET : «VIVRE ET SE DÉVE- LOPPER AVEC LE FLEUVE »}

Le projet intéresse la Loire et ses abords sur un linéaire d'environ $5 \mathrm{~km}$ qui s'étend entre le pont de la RD 103 à l'aval et le pont du Cévenol à l'amont, les actions les plus importantes étant concentrées sur le secteur urbain en amont de la RN 88.

\subsection{Les aménagements pour lutter contre les crues}

\subsubsection{L'aménagement du lit mineur}

La suppression de points hauts du lit mineur constitués en grande partie d'affleurements rocheux intéresse plus particulièrement la zone centrale située de part et d'autre du pont de Gallard où le décaissement maximum atteint $2,50 \mathrm{~m}$. Le volume de matériaux à extraire correspond à $250000 \mathrm{~m}^{3}$.

Le recalibrage latéral du lit mineur consiste à redonner à la Loire une section régulière qui avait été réduite soit par remblaiement soit par sédimentation naturelle. La pérennité de cette section est maintenue par un profil d'équilibre qui tend à uniformiser les vitesses d'écoulement.

Ces élargissements restent toutefois localisés et représentent un volume de matériaux à extraire de $110000 \mathrm{~m}^{3}$.

\subsubsection{L'aménagement du champ majeur}

Il concerne la création de risbermes par rabaissement des terrains et uniformisation de leur seuil de débordement. Le volume de matériaux à extraire est de $130000 \mathrm{~m}^{3}$. On accroît ainsi la capacité de transit du fleuve en lui associant une bande longitudinale d'écoulement continue de largeur uniforme sur laquelle tout obstacle a été enlevé.

A ce titre, les bâtiments de 3 usines sont partiellement démolis, les bâtiments restants étant réhabilités, les remblais routiers de l'ouvrage de franchissement de la RN 88 sont percés d'ouvrages de décharges supplémentaires.
Toujours pour améliorer l'écoulement, on réalise la sup pression d'ouvrages et un déboisage sélectif notamment sur la partie aval sur près de $2 \mathrm{~km}$ (fig. 2).

\subsection{Les aménagements liés à la revalorisation du site}

\subsubsection{L'aménagement du fleuve}

\section{- La création de plans d'eau}

La section du lit mineur étant agrandie et rabaissée; il convenait sur le plan esthétique de rehausser les niveaux d'eau en période d'étiage.

Pour ce faire, trois seuils mobiles ont été prévus sur le parcours urbain et permettent le maintien de plans d'eau qui, outre l'aspect esthétique, constituent une opportunité pour les loisirs.

Chaque seuil mobile comporte 4 clapets activés par des vérins. Les seuils amont et aval ont une hauteur de $1,80 \mathrm{~m}$. Le seuil central correspond à une hauteur de $4,20 \mathrm{~m}$.

Le système de régulation des clapets asservi au niveau amont assure le maintien d'un niveau constant en période normale et pour les faibles crues.

Pour les crues plus importantes, le dispositif de régulation des clapets associé au système de prévision des crues permet de les effacer complètement en libérant la totalité de la section d'écoulement.

- La création d'embarcadère et de plages liés aux plans d'eau amont et intermédiaire.

\subsubsection{L'aménagement des abords du fleuve}

Aménagements de cheminement pour longer et accéder au fleuve. Il s'agit de pistes cyclables sur tout le linéaire, de voies piétonnes, associées à des belvédères qui dominent les plans d'eau.

- Aménagements pour traverser le fleuve avec la réalisation d'une passerelle en amont, d'un encorbellement du Pont de Gallard et du prolongement du Pont de la Chartreuse.

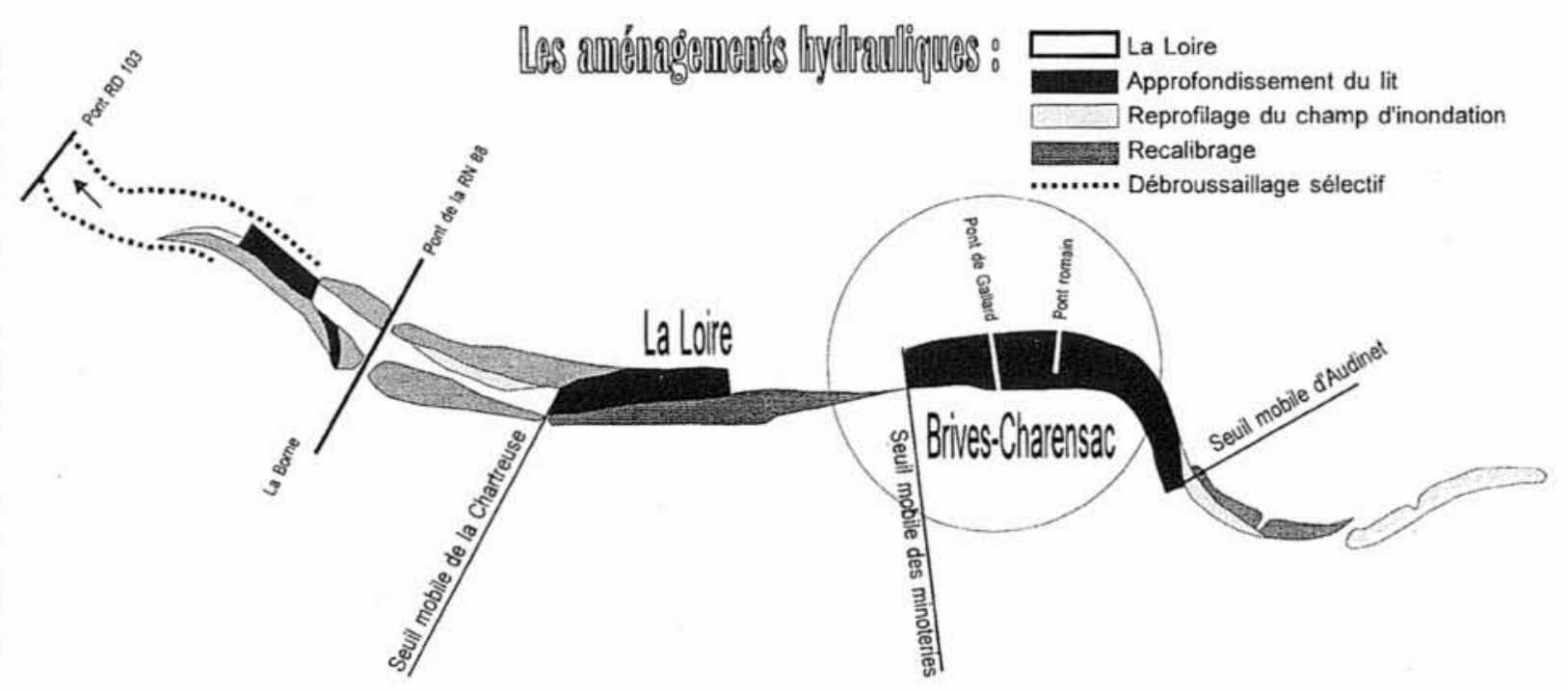

2. Les aménagements hydrauliques près de Brives-Charensac. 


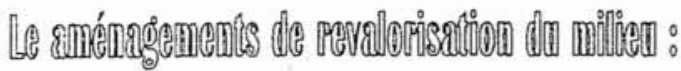

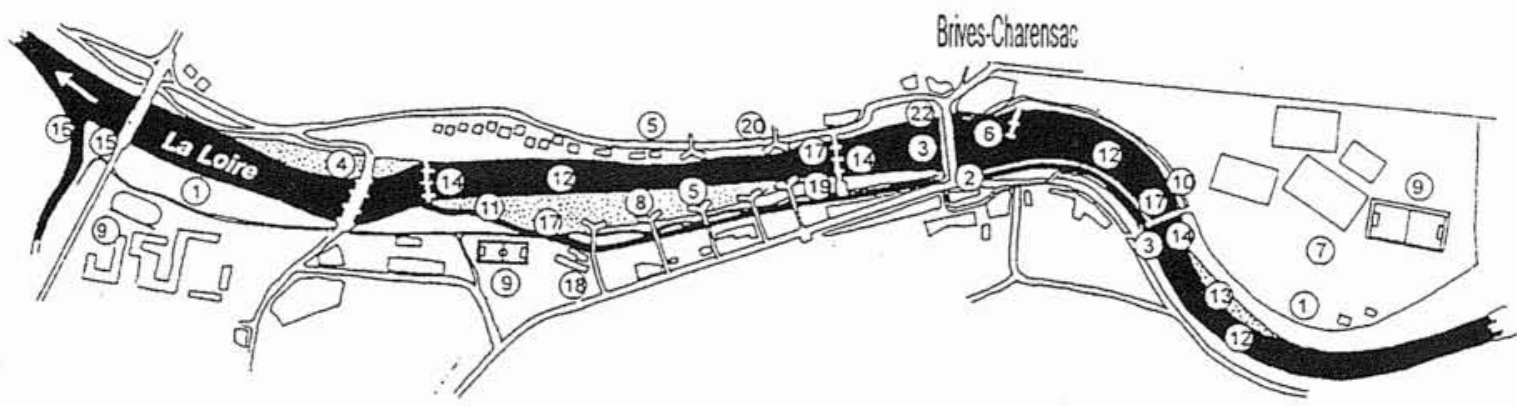

\section{Parcourir le fleuve}

Longer le fleuve

1- Création d'une piste cyclable.

2 - Aménagement et prolongation du Canal de la Dunière.

Traverser le fleuve

3-Réalisation d'une passerelle sur le seuil d'Audinet.

4 - Réalisation d'une arche de pont fusible sur le pont de la Chartreuse.

Accéder au fleuve

5- Création de 7 "balcons" sur la Loire.

6- Réhabilitation du Pont Vieux.
Se détendre avec le fleuve

7 - Extension du camping d'Audinet.

8 - Création d'aires de jeux et de pique-nique.

9 - Création et modification de terrains de sport.

10 - Création d'un embarcadère.

II - Parcours d'initiation au kayak.

12 - Création de 3 plans deau.

13 - Aménagement d'une plage.
Réguler le fleuve

14 - Création de 3 seuils mobiles.

15 - Réalisation d'un ouvrage de décharge sous la RN 88.

16 - Aménagement de la confluence de la Borne.

17 - Création de 3 passes à poissons.
Vivre auprès du fleuve

18 - Restructuration des bâtiments et des abords de la Dunière.

19 - Restructuration des bâtiments et des abords de Celle.

20 - Restructuration des bâtiments et des abords de la minoterie Sabarot.

21 - Place du $8 \mathrm{Mai}$ : Aménagement d'une entrée de ville.

22 - Extension du Square Jean Moulin.

3. Les aménagements de revalorisation du milieu le long de la Loire près de Brives-Charensac.

\subsubsection{L'aménagement urbain}

Il comprend, notamment, la restructuration des 3 usines, l'aménagement de places, de squares, d'un camping.

\subsection{L'aménagement lié à la valorisation et la protection du milieu}

- L'ancien canal d'alimentation des usines en rive gauche du fleuve est aménagé pour assurer une continuité entre le plan d'eau amont et l'aval du dernier seuil. Ce canal offre un double usage en permettant la remontée du poisson et la pratique d'activités ludiques ou sportives (fig. 3).

\section{IES TRAVAUX}

Le déroulement des travaux et ceux notamment relatifs au lit mineur sont prévus en période de faible hydraulicité dans le souci de garantir la sécurité des riverains contre les crues et de minimiser les nuisances liées à la réalisation des travaux.

Les travaux d'amélioration des écoulements seront tous réalisés avant fin 1996. Les travaux de revalorisation du site se dérouleront jusqu'à fin 1997 compte tenu des délais nécessaires pour la délocalisation de certaines entreprises. De même, la pose des clapets mobiles ne pourra être terminée que durant la période d'étiage 1997.

L'extraction des matériaux meubles et rippables sont effectués par les engins classiques de terrassement. Les matériaux rocheux essentiellement constitués d'arkose et de granit sont fracturés suivant les techniques de minage par micro-charges compte tenu de l'environnement urbain.

Au droit des sites sensibles et notamment au pied du pont de Gallard et de l'ancien Pont Romain, la fracturation de la roche met en œuvre des techniques évitant toute propagation d'onde de choc.
Dans le cadre de ces travaux, une cellule de suivi de l'environnement a été créée. Elle regroupe des administrations, collectivités et associations animées par un coordonnateur. Cette démarche a pour but de limiter durant la période de travaux les nuisances liées à l'environnement et au cadre de vie des riverains.

Le montant des travaux correspond à un coût d'objectif de 280 millions de Francs.

Ce montant se décompose approximativement à parts égales entre les travaux et les acquisitions foncières. Pour ce qui concerne les travaux, leur montant est équiréparti entre la protection contre les crues et la revalorisation du site.

\section{VI. - CONCLUSION}

Au cours du $\mathrm{XIX}^{\mathrm{c}}$ siècle, l'essor industriel a favorisé l'implantation des usines au bord de l'eau et la ville a souvent "tourné le dos " à la rivière parce que celle-ci constituait un milieu hostile par ses débordements intempestifs et dommageables.

Les développements urbains liés à la pression urbanistique de la seconde moitié de notre siècle ont vu progressivement la colonisation de ces milieux par l'urbanisation des zones inondables, la rivière restant souvent l'élément hostile qu'il convenait de corseter, de canaliser et même d'ignorer.

Les événements récents en France et dans le monde ont mis en évidence les méfaits de cette stratégie d'aménagement et une nouvelle politique plus douce et mieux adaptée visant à « apprendre à vivre avec les fleuves » est née,

Pour que cette politique ne relève pas du domaine de l'utopie, il fallait créer des liens nouveaux et forts entre l'homme et le fleuve, trouver le bon compromis entre revalorisation des sites et protection humaine. C'est sur ces bases nouvelles que repose le projet d'aménagement de la Loire et de ses abords dans la traversée de Brives-Charensac. 